\title{
Hipnosis anestésica inducida por profesional de Enfermería. Reporte de un caso
}

\section{Nursing staff induced anesthetic hypnosis: A case report}

\section{Hipnose anestésica induzida por profissional de Enfermagem. Relatório de um caso}

\author{
E. Álvarez ${ }^{\text {a1* }}$, N. Medina ${ }^{\text {b2 }}$ \\ ORCID: \\ a $0000-0002-3748-8631$ \\ ${ }^{\mathrm{b}} 0000-0002-0205-6353$ \\ ${ }^{1}$ Carrera de Enfermería. Departamento de Ciencias Clínicas y Pre Clínicas, Facultad de Medicina. Universidad \\ Católica de la Santísima Concepción, Concepción, Chile \\ ${ }^{2}$ Dirección de Apoyo a los Estudiantes. Universidad Católica de la Santísima Concepción, Concepción, Chile
}

Recibido: 23 mayo 2018

Aceptado: 13 enero 2019

\section{Resumen}

Introducción: La hipnosis anestésica puede ser un recurso útil para procedimientos dolorosos.

Desarrollo: Se reporta caso de una joven que requiere exodoncia de terceros molares, con uso de hipnosis como único anestésico. El proceso de enfermería inicia con la valoración según patrones funcionales, impresiona lo sana que se encuentra, sin antecedentes mórbidos relevantes ni contraindicación para anestesia química. Se selecciona diagnóstico NANDA de Disposición para mejorar el confort. Dentro del plan se incorporan el NIC 5920 Hipnosis y 5922 Facilitar la autohipnosis, con buena evolución según lo planificado.

Resultados: La extracción con hipnosis anestésica se desarrolló de forma similar a la cirugía con anestesia química, la cual se realizó sin dolor. En posoperatorio, paciente practica autohipnosis, sin necesitar analgésicos y evolucionó favorablemente.

Discusión: La hipnosis requiere mayor evidencia científica, pero su uso es prometedor. Se constataron los beneficios de la hipnosis en el abordaje del dolor.

Conclusión: La hipnosis anestésica, fue útil como intervención de enfermería en cirugía dental. La autohipnosis contribuyó al buen resultado. El profesional de Enfermería entrenado en hipnosis está en 
condiciones de incorporarla en la gestión del cuidado. El caso resulta interesante, dado que la paciente opta por la hipnosis, sin contraindicación para anestesia química, ya que no se encontró otro reporte de hipnoanestesia inducida por profesional de Enfermería, como anestésico único. Anecdóticamente, durante la filmación del procedimiento, una periodista entra espontáneamente en trance, por lo que requiere intervención del profesional de Enfermería para salir de él.

Palabras clave: Hipnosis; atención de enfermería; hipnosis anestésica; Chile.

\section{Abstract}

Introduction: Anesthetic hypnosis can be a useful resource to address painful procedures.

Development: The case is of a young female patient requiring third-molars exodontia who underwent it under anesthetic hypnosis. The nursing process began with the assessment according to functional patterns. The patient was found healthy and without relevant morbid antecedents nor drug-anesthesia contraindications. A comfort improving disposition NANDA diagnosis was selected. NIC 5922 and 5920 were incorporated to the intervention plan.

Results: The extraction under anesthetic hypnosis was performed in a similar form in comparison to a drug-anesthesia extraction procedure. During the post operatory stage, the patient used self-hypnosis and responded favorably without analgesics.

Discussion: While hypnosis requires more scientific evidence, its use appears to be promising. In this case, diverse benefits from using hypnosis to address a painful procedure were acknowledged.

Conclusion: Anesthetic hypnosis proved being helpful in a dental-surgery related nursing intervention. Self-hypnosis also contributed to the favorable outcome. A nursing professional who is well trained in hypnosis can incorporate this kind of procedure into her care management approach. This particular case was interesting because the patient chose hypnosis over drug anesthesia, and no other report on a nursing staff induced anesthetic hypnosis was found. Anecdotally, during the filming of this procedure, a journalist suffered an emotional trance which required immediate nursing intervention.

Keywords: Hypnosis; nursing care; hypnotic anesthesia; Chile.

\section{Resumo}

Introdução: A hipnose anestésica pode ser um recurso útil para procedimentos dolorosos.

Desenvolvimento: Informa-se o caso de uma garota que requer exodontia de terceiros molares, com uso de hipnoses como único anestésico. O processo de enfermagem inicia com a valoração segundo padrões funcionais, impressiona o saudável que se encontra, sem antecedentes mórbidos relevantes nem contraindicação para anestesia química. Seleciona-se diagnóstico NANDA de Disposição para melhorar o conforto. Dentro do plano se incorporam o NIC 5920 Hipnose e 5922 Facilitar a auto-hipnose, com boa evolução segundo o planejado.

Resultados: A extração com hipnose anestésica desenvolveu-se de forma similar à cirurgia com anestesia química, a qual se realizou sem dor. Em pós-operatório, o paciente prática auto-hipnose, sem precisar analgésicos e evolucionou favoravelmente.

Discussão: A hipnose requer maior evidencia científica, mas seu uso é promissor. Verificaram os benefícios da hipnose na abordagem da dor.

Conclusão: A hipnose anestésica foi útil como intervenção de enfermagem em cirurgia dental. A auto-hipnose contribuiu para o bom resultado. O profissional de Enfermagem treinado em hipnose está em condições de incorporá-la na gestão do cuidado. O caso resulta interessante, dado que a paciente opta pela hipnose, sem contraindicação para anestesia química, já que não se encontrou outro relatório de hipnoanestesia induzida por profissional de Enfermagem, como anestésico único. Anedoticamente, durante a filmagem do procedimento, uma jornalista entrou espontaneamente em transe, pelo qual se requereu intervenção do profissional de Enfermagem para sair dele.

Palavras chave: Hipnose; atenção de enfermagem; hipnose anestésica; Chile. 


\section{Introducción}

El manejo del dolor es una de las aplicaciones más interesantes de la hipnosis ${ }^{1}$. A pesar de sus potenciales beneficios, su oferta a los pacientes es limitada por los mitos y prejuicios que existen incluso en el personal de salud ${ }^{2}$, y por la poca disponibilidad de profesionales que manejen la técnica ${ }^{3}$.

Como intervención de Enfermería, la hipnosis es considerada como la acción de ayudar al paciente a conseguir un estado de concentración atenta y centrada con suspensión parcial de la conciencia periférica, para crear cambios de la sensibilidad, los pensamientos o la conducta ${ }^{4}$. En la literatura, puede encontrarse que se usa el término hipnosis o la expresión "hétero hipnosis", para referirse a la situación en que una persona es hipnotizada con la ayuda de un tercero, para este caso el profesional de enfermería. Por otra parte, se utiliza el término "autohipnosis", para referirse al caso en que la persona es entrenada en controlar el uso de un estado hipnótico "autoinducido”, para un beneficio terapéutico ${ }^{4}$.

Para Zahourek ${ }^{5}$, enfermera, la hipnosis puede comprenderse como un estado alterado de conciencia; donde las personas tienden a ser más receptivas a nuevas ideas o sugestiones. Coincide con otros autores en que es un fenómeno psicofisiológico normal, relacionado con un nivel de disociación de la conciencia. En este estado especial de conciencia o trance hipnótico, se evidencia, entre otros fenómenos hipnóticos, la hipnosis anestésica o hipnoanestesia, cuyo rol en cirugía fue preponderante, previo al surgimiento de los anestésicos químicos ${ }^{6}$.

Aunque la revisión de la literatura sugiere más estudios que apoyen la evidencia prometedora de la hipnosis en el ámbito de la cirugía y procedimientos médicos dolorosos, esta da cuenta de sus beneficios sobre la ansiedad, el dolor, el uso de medicamentos, parámetros fisiológicos, recuperación y el tiempo del procedimiento quirúrgico ${ }^{7-9}$. Según la revisión de $\mathrm{Facco}^{8}$, la hipnosis puede modificar la actividad de la corteza prefrontal y de la neuromatriz del dolor, por lo tanto aumenta su umbral hasta el nivel de la anestesia quirúrgica, también previene la respuesta cardiovascular al dolor y protege al paciente de los efectos del estrés, destaca que su aplicación no requiere medicamentos ni equipos, no se asocia a eventos adversos probados y es de bajo costo.

Asimismo, según la revisión de Facco, la hipnosis puede ser utilizada como único anestésico, en cirugía menor y maniobras invasivas, como coadyuvante de la anestesia farmacológica local o general. Precisamente, en el campo de la odontología, se ha descrito su utilidad como anestésico único ${ }^{10}$ o combinada con anestesia farmacológica ${ }^{11}$; también en dolor clínico, habitualmente por problemas con la anestesia química ${ }^{12}$ y en dolor experimental ${ }^{13}$. En el ámbito de Enfermería, la hipnosis ha sido considerada como intervención enfermera con el NIC $5920^{4}$, refiriéndose por Montilla y Amador ${ }^{3}$ los siguientes usos encontrados dentro de la literatura disciplinar, en una revisión de 5 años publicada en 2017: mejora del síndrome de intestino irritable, disminución del dolor y estrés, mejora en trabajo del parto, y en menor medida control de la frecuencia cardiaca, de la frecuencia respiratoria y de la ansiedad. En $2016^{14}$, se publica un estudio comparativo con pacientes operados de cataratas, en el cual la hipnosis es administrada por una profesional de enfermería anestesista, reportándose que no hubo diferencias en los parámetros fisiológicos entre el grupo hipnotizado y el grupo control, fue mayor el uso de medicamentos intraoperatorios en el grupo control, y satisfacción por el uso de la técnica en los hipnotizados.

Este artículo, presenta la aplicación del proceso de enfermería en el caso de una paciente que requería la exodoncia de dos terceros molares, para tratamiento de ortodoncia y decide usar hipnosis como anestésico único. Los objetivos del artículo son: exponer un enfoque práctico y novedoso para el abordaje del dolor asociado a un procedimiento quirúrgico odontológico, por medio de la hipnosis anestésica inducida por un profesional de Enfermería, lo cual no fue encontrado en la revisión de la literatura, por lo anterior, motivar a los profesionales de enfermería a considerar la hipnosis dentro de su repertorio de competencias para la gestión del cuidado.

Es interesante que la revisión de la literatura no arrojara otro informe de hipnosis anestésica inducida por Enfermera/o, como anestésico único en cirugía y que la paciente optara por su uso a pesar de no tener contraindicación para anestesia química. En este reporte, se presenta el plan de cuidados y el 
proceso realizado para lograr la hipnosis anestésica. El procedimiento fue llevado a cabo con éxito, sin inconvenientes y con una evolución favorable de la paciente.

\section{Desarrollo}

\section{Descripción del caso}

Se presenta el caso de una mujer de 20 años, sana y autovalente con indicación de exodoncia de terceros molares por necesidad de tratamiento ortodóncico. Durante el taller de relajación en el que participa como parte del currículo de su carrera, logra trance profundo e hipnoanestesia; luego del cual, solicita al Enfermero especialista, apoyarla en el uso de hipnosis anestésica para este procedimiento. La paciente no presenta contraindicación a la anestesia química. Se cita a entrevista y se coordina evaluación odontológica.

El abordaje del caso se realiza a través del Proceso de enfermería, exponiéndose la sistematización de los cuidados programados para la creación del trance, instalación de la hipnosis anestésica, apoyo en el intraoperatorio y seguimiento realizado con el fin de abordar la necesidad de exodoncia de la paciente.

\section{Valoración}

La valoración se realizó conforme a la guía de valoración del adulto según patrones funcionales ${ }^{15}$.

1. Patrón percepción de la salud-manejo de la salud

Paciente no presenta enfermedades crónicas. Fue operada a los tres años por un cuadro de peritonitis del cual se recuperó sin problemas. Refiere haber sido diagnosticada con gastritis crónica y úlcera gástrica en 2007, tratada inicialmente con Omeprazol y luego con Lanzoprazol 1 al día por 3 meses, que ceso con este tratamiento. Sin otros antecedentes mórbidos de importancia. Buen estado general de salud. Sin alergias conocidas. No presenta consumo problemático de sustancias. Actualmente, requiere la extracción de terceros molares para finalizar proceso de ortodoncia.

2. Patrón nutricional-metabólico

Impresiona eutrófica, no sigue una dieta específica, pero come variado. No presenta alteración en masticación ni en deglución.

3. Patrón eliminación

No refiere problemas de eliminación.

4. Patrón actividad-ejercicio

Independiente, con un nivel de completo autocuidado en todas sus capacidades. Sin problemas de movilidad. Practica ejercicio físico sin limitaciones.

5. Patrón Sueño-descanso

Refiere no tener problemas para conciliar el sueño. Duerme seis horas por día sin necesidad de siesta. Despierta con energía y sintiéndose descansada.

6. Patrón cognitivo-perceptual

Consciente, lúcida, orientada en tiempo, espacio y persona. Refiere presentar audición y visión normales. Su lenguaje es coherente y se comunica sin inconvenientes. Mantiene buena concentración. Tiene niveles de aprendizaje que le permiten desempeñarse exitosamente en sus estudios universitarios. No refiere malestar o dolor al momento de la entrevista, ni en la vida diaria.

7. Patrón autopercepción-autoconcepto

Se describe como una persona segura, positiva, con ánimo y que se siente bien consigo misma.

8. Patrón rol-relaciones

En la actualidad vive con su abuela materna y un tío, pero conserva relaciones muy unidas con sus padres y hermana menor. Se integra sin problemas en los grupos e interactúa bien con las demás personas, mantiene relaciones sociales saludables a nivel familiar, laboral, de pareja y de amistad, asume incluso rol de representación de pares. 


\section{Patrón sexualidad-reproducción}

Paciente nulípara, sin antecedentes de problemas ginecológicos.

10. Patrón adaptación-tolerancia al estrés

Paciente refiere estar bien adaptada en los ambientes en los que se desenvuelve: familiar, académico y laboral. Su desempeño académico es bueno, supera exitosamente los períodos de mayor exigencia. En la etapa escolar experimentó Bullying y aunque en la actualidad el recuerdo de la situación le hace experimentar sentimientos de tristeza, refiere haberlo superado sin terapia, sólo con cambio de establecimiento educacional.

\section{Patrón valores-creencias}

Entre sus planes de futuro importantes están el terminar su carrera, trabajar, formar una familia con su novio y tener hijos. Refiere que Dios es importante en su vida, y la fe cuando surgen dificultades.

A la evaluación odontológica, paciente en condiciones para la exodoncia. Se indica profilaxis antibiótica, se educa, además se le explica que puede solicitar anestesia química en cualquier momento. Se resuelven dudas y se entrega material escrito al respecto. Se acuerda que de funcionar la hipnosis anestésica durante la sindesmotomía o sección de ligamentos articulares, se continuará según lo programado.

\section{Tabla 1. Esquema Plan de cuidados}

\begin{tabular}{|c|c|c|c|}
\hline 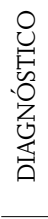 & \multicolumn{3}{|c|}{$\begin{array}{l}\text { DOMINIO 12: Confort } \\
\text { NANDA [00183] } \\
\text { Disposición para mejorar el confort r/c próxima exposición a exodoncia m/p por la paciente verbalmente } \\
\text { Características definitorias: } \\
\text { Expresa deseos de aumentar el confort } \\
\text { Expresa deseos de aumentar la relajación }\end{array}$} \\
\hline & ETIQUETA & $\begin{array}{l}\text { NOC [2010] Estado de comodidad: Física } \\
\text { NOC [1843] Conocimiento: Manejo del dolor }\end{array}$ & \\
\hline & INDICADORES & $\begin{array}{l}\text { [201001] Control de síntomas (Dolor) } \\
\text { (De Gravemente comprometido a No comprometido) }\end{array}$ & $\begin{array}{l}\text { 5. No comprometido } \\
\text { Mantener en No comprometido }\end{array}$ \\
\hline & & $\begin{array}{l}\text { [184303] Estrategias para controlar el } \\
\text { dolor. (Hipnosis y autohipnosis) } \\
\text { (De ningún conocimiento a Conocimiento extenso) }\end{array}$ & $\begin{array}{l}\text { 2. Conocimiento escaso } \\
\text { Aumentar a Conocimiento sustancial }\end{array}$ \\
\hline 学 & \multicolumn{3}{|c|}{$\begin{array}{l}\text { [5920] Hipnosis } \\
\text { [5922] Facilitar la autohipnosis }\end{array}$} \\
\hline 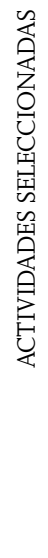 & \multicolumn{3}{|c|}{$\begin{array}{l}\text { Realizar la anamnesis del problema que se va a tratar mediante hipnosis. } \\
\text { Corregir los mitos y conceptos equivocados de la hipnosis. } \\
\text { Determinar los objetivos de la hipnosis con el paciente. } \\
\text { Comentar con el paciente la sugestión hipnótica que se usará antes de la inducción. } \\
\text { Se asegura a la paciente que, en caso de requerirlo, podrá solicitar anestesia } \\
\text { química en cualquier momento del procedimiento. } \\
\text { Explicar que el nivel del trance no es importante para que la hipnosis tenga éxito. } \\
\text { Determinar la receptividad del paciente al empleo de la hipnosis (Sugestionabilidad). } \\
\text { Establecer rapport. (Relación de confianza con el paciente). } \\
\text { Usar procedimiento de inducción hipnótica. } \\
\text { Ayudar al paciente a generar su "lugar seguro" utilizando la imaginación guiada. } \\
\text { Ayudar al paciente a identificar técnicas de profundización del trance. } \\
\text { Facilitar la inducción rápida por medio del "signo-señal" (estímulo condicionado). } \\
\text { Entrenar al paciente en el uso de las sugestiones específicas para crear la hipnoanestesia. } \\
\text { Dar sugestiones específicas para la práctica posterior de autohipnosis. } \\
\text { Facilitar la salida del paciente del trance contando hasta un número prefijado. }\end{array}$} \\
\hline & \multicolumn{3}{|c|}{$\begin{array}{l}\text { Indicar autohipnosis como técnica complementaria al trabajo con hipnoanestesia. } \\
\text { Enseñar al paciente el concepto e importancia de la autohipnosis como modalidad terapéutica. } \\
\text { Animar al paciente a dominar la autohipnosis practicándola. }\end{array}$} \\
\hline
\end{tabular}

Diagnóstico de Enfermería según taxonomía NANDA [00183]:

Disposición para mejorar el confort relacionado con próxima exposición a exodoncia manifestado verbalmente por la paciente ${ }^{4}$. 
Características definitorias:

Expresa deseos de aumentar el confort

Expresa deseos de aumentar la relajación

Se espera preparar a la paciente para enfrentar exodoncia con mejor disposición, sin dolor, mantener el autocontrol y de forma relajada.

Etiquetas Criterios de Resultados $\mathrm{NOC}^{4}$ :

NOC [2010] Estado de comodidad: Física

NOC [1843] Conocimiento: Manejo del dolor

Indicadores ${ }^{4}$ :

[201001] Control de síntomas (Dolor). Se espera mantener en No comprometido intra y post quirúrgico. Para asegurar manejo del dolor, la paciente puede solicitar anestesia química en cualquier momento. [184303] Estrategias para controlar el dolor (Hipnosis y autohipnosis). Se espera aumentar de conocimiento escaso a conocimiento sustancial. Iniciar abordando mitos, prejuicios, proceso y resolver dudas. Se explica la forma en que el paciente puede colaborar. Se enseña autohipnosis.

Intervenciones $\mathrm{NIC}^{4}$ :

[5920] Hipnosis. Ayudar al paciente a conseguir un estado de concentración atenta y centrada con suspensión parcial de la conciencia periférica, para crear cambios de la sensibilidad, los pensamientos o la conducta.

[5922] Facilitar la autohipnosis. Enseñar y controlar el uso de un estado hipnótico autoinducido para un beneficio terapéutico.

Ejecución del Cuidado

Hipnosis. Sesión 1.

Se realiza valoración. Se abordan detalles del procedimiento, mitos y prejuicios. Se resuelven dudas y se entrega material escrito, lo cual es muy importante para establecer la relación de confianza entre el enfermero y la paciente, así como respetar su derecho a conocer el tratamiento al que va a ser sometida.

Se induce trance por héterohipnosis cara a cara, sin uso de grabaciones, ni de libreto o guión; debido a que el profesional de enfermería prefirió utilizar una inducción adaptada a la paciente, en este se incorporan sus preferencias e ideas personales acerca de lo que para ella es relajante y tranquilizador. Se establece el "lugar seguro” pidiéndole que busque en su mente, un sitio donde experimente protección, con el fin de aumentar la confianza y generar un espacio de seguridad donde ir con su mente, en caso de que durante el procedimiento la persona se contacte con alguna experiencia traumática real o imaginada. Se dan sugestiones de profundización, se logra relajación general, disminución de frecuencia respiratoria, catalepsia palpebral espontánea y catalepsia del brazo sugerida.

Se instala el cierre de ojos, respiración relajada y contacto con lugar seguro como "signo-señal" lo cual corresponde a un estímulo condicionado que se usa para facilitar el ingreso al trance. Sale del trance, asociándolo a la sensación de "estar dentro de una burbuja”. Finaliza ejercitando entrada y salida del trance sin problemas de forma autónoma, lo cual le sirve como entrenamiento en autohipnosis, la que se le indica debe practicar una vez al día, con la finalidad de potenciar sus resultados con el uso de la hipnosis.

Hipnosis. Sesión 2.

Informa práctica de autohipnosis exitosa. Se discute el consentimiento informado para realizar el procedimiento y grabación en video, posteriormente lo firma.

Aunque, según la literatura9 ${ }^{9}$, puede haber buenos resultados, de acuerdo con la hipnosis independiente del grado de susceptibilidad hipnótica de las personas, se sugiere que sea medida, lo cual en este caso se hizo con la escala de Stanford forma C versión mexicana ${ }^{16}$, elegida porque es ampliamente usada y es 
una versión en español validada. Obtiene 10 puntos, para alta susceptibilidad. Cabe mencionar que, en el ítem de regresión de edad, recordó el episodio de bullying que sufrió en primaria y llora; lo cual cedió al sugerirle que se imaginara a esa edad, en un lugar más agradable. Posteriormente, se le consulta por esta experiencia, en la que refiere "sentí una emoción, la cual se fue, de manera inmediata al cambiar mentalmente de lugar. Para mí es un tema superado”. Luego de la escala, y con el propósito de evaluar su práctica personal, se le pide entrar en el estado hipnótico de manera autónoma, lo que realiza sin inconvenientes.

Una vez que está en hipnosis, se prueba con éxito la "anestesia en guante", técnica que consiste en crear hipnoanestesia en una mano para luego traspasarla a otra parte del cuerpo. Se comienza, pidiéndole concentrar su atención en la mano derecha y crear mentalmente en ella sensaciones de anestesia dental. Se refuerza con sugestiones de frío e insensibilidad. Logrado el efecto sugerido, se desinfecta el dorso de la mano y se forma un pliegue con la piel, traspasándola de lado a lado con aguja hipodérmica $\mathrm{n}^{\circ}$ 21; sin evidencias de dolor. Se retira aguja. Luego, se le pide traspasar la hipnosis anestésica a la mano izquierda y descansar la derecha sobre ella. Para comprobar el efecto se puncionó la piel con aguja 21. Se retira la aguja y se le indica traspasar la hipnoanestesia al área de la cara que será intervenida, se evaluó la intensidad de la anestesia obtenida de 0 a 10. Informa 7. Para que logre controlar el grado de anestesia producida, se le sugiere aumentarla e imaginar que lo hace moviendo una perilla. Lo realiza sin problemas. Se dan sugestiones de tumefacción para producir control del sangramiento y disminuir salivación. Sale del trance, refiere lograr fácilmente la hipnoanestesia, su traspaso y aumento de potencia. Reporta dolor 0 en escala EVA en todo momento. Se indica practicar autohipnosis y la hipnosis anestésica.

Primera extracción.

La paciente es recibida por el Enfermero, se entrevista para video. Se le pide entrar en el estado y que "sin salir del trance" abra los ojos y vaya a la clínica odontológica. Ingresa, se acomoda en el sillón dental y cierra los ojos. Genera hipnoanestesia en la zona operatoria y la intensifica en 30 segundos.

Se inicia sindesmotomía; sin signos de dolor clínicos, ni verbales. Continúa intervención sin incidentes, con una pausa a petición de la paciente, para aumentar la potencia anestésica de manera autónoma, lo cual realiza con éxito, e imagina que lo hace al mover una perilla. El Enfermero la apoya con sugestiones para disminuir sangramiento y salivación. Durante el procedimiento la paciente permanece relajada, sin movimientos y atenta a colaborar con las instrucciones del dentista. Se finaliza en una hora.

La paciente sale del trance relajada, describe haberse sentido segura, consciente y sin dificultad para seguir las instrucciones. Reportó dolor cero en escala EVA en todo momento, explicó el porqué de la pausa solicitada, al sentir una molestia que controló intensificando la hipnoanestesia de forma autónoma.

Para el odontólogo la única diferencia del procedimiento fue un poco más de sangrado con la hipnoanestesia, dado que la anestesia química posee un vasoconstrictor. Prescribe usar un analgésico-antiinflamatorio, en caso necesario. El Enfermero indica autohipnosis diaria, y usar sugestión de tumefacción para apoyar control del dolor y la inflamación.

Segunda extracción.

Una semana después, se replica procedimiento sin inconvenientes, observándose mayor autonomía y rapidez. Se repiten indicaciones postoperatorias.

\section{Resultados}

Posterior a ambas exodoncias, se realiza seguimiento de la paciente, quien informa no haber experimentado dolor ni inflamación; con sólo la autohipnosis, sin medicamentos. Sin infección. Una semana después se encuentra recuperada, satisfecha de haber usado hipnosis.

El problema principal de la paciente, que consistía en la necesidad de realizarse la exodoncia, se solucionó con el procedimiento quirúrgico sin uso de anestesia química, mantuvo su disposición para mejorar el confort durante todo el proceso, según el diagnóstico NANDA planteado. 
Acerca del NOC [2010] Estado de comodidad: física, según el indicador 201001 Control de síntomas (Dolor), no requirió anestesia química y presentó buena evolución; sin comprometer el control del dolor.

Respecto del NOC [1843] Conocimiento: Manejo del dolor, según el indicador 184303 Estrategias para controlar el dolor. (Hipnosis y autohipnosis), la paciente aumentó su conocimiento a sustancial, evidenciándolo en la práctica diaria de la autohipnosis y de las destrezas para controlar el grado de la hipnoanestesia lo que le permitió su uso sin problemas durante el procedimiento.

Las intervenciones de enfermería escogidas para lograr el trance y la hipnosis anestésica contribuyeron, positivamente al logro del éxito terapéutico, no se presentaron limitaciones durante la intervención, procediéndose según lo planificado y sin necesidad de hacer cambios durante la ejecución.

Anecdóticamente, durante la filmación una periodista entró en trance de forma espontánea, (aunque no hubo inducción hipnótica, dado que la paciente entra sola en el estado). El Enfermero apoya su salida del estado, sin inconvenientes.

\section{Discusión}

La hipnosis requiere mayor evidencia científica, especialmente en el ámbito de Enfermería. Se coincide con Tefikow y colaboradores ${ }^{7}$, en que los resultados de la aplicación de la hipnosis son promisorios.

En coincidencia con lo encontrado en la literatura ${ }^{7-9}$ en el caso relatado, se observaron beneficios de la hipnosis sobre la ansiedad, el dolor, el uso de medicamentos y la recuperación post operatoria. Asimismo, pudo comprobarse lo señalado por $\mathrm{Facco}^{8}$, en cuanto a que, la paciente pudo aumentar su umbral de dolor hasta el nivel de la anestesia quirúrgica sin necesidad de fármacos. El procedimiento hipnótico fue llevado íntegramente por el profesional de Enfermería, el manejo del dolor es una de las principales aplicaciones de la hipnosis encontrada en una revisión de la literatura de enfermería, publicada en $2017^{3}$. A diferencia de lo reportado en Francia ${ }^{14}$, en que una enfermera anestesista usó hipnosis en cirugía de cataratas, en este caso no se utilizó medicación, además se realiza una exposición del proceso hipnótico y se presenta el plan de cuidados, lo cual no aparece en el artículo francés.

Muchos procedimientos clínicos son dolorosos, y los pacientes podrían beneficiarse, si se les ofreciera usar hipnosis. Se espera que este caso contribuya al interés de los profesionales de Enfermería por la hipnosis como intervención enfermera, en su rol independiente o en colaboración con otros profesionales.

\section{Conclusiones}

- La hipnosis anestésica, ofreció un enfoque novedoso para abordar el dolor durante la exodoncia de terceros molares, lo que permitió a la paciente, afrontar exitosamente el procedimiento, sin usar fármacos para el control del dolor, con una evolución óptima.

- La autohipnosis, contribuyó a la autonomía en el proceso hipnótico y al control del dolor e inflamación post operatorios.

- El profesional de Enfermería con un entrenamiento específico está en condiciones de incorporar la hipnosis clínica dentro de la gestión del cuidado.

- Aunque en este caso no hubo contraindicación para la anestesia química, se entiende la mayor importancia de la hipnosis en personas que si la presentan.

\section{Responsabilidades éticas}

Protección de personas y animales. En esta investigación, no se han realizado experimentos en seres humanos ni en animales.

Confidencialidad de los datos. Los autores declaran que en este artículo no aparecen datos sensibles sobre la paciente.

Derecho a la privacidad y consentimiento informado. La paciente ha firmado el consentimiento informado para la publicación del artículo, el que obra en poder del autor de correspondencia 
Financiamiento. Ninguno.

Conflicto de intereses. Los autores declaran no tener conflicto de intereses.

\section{Referencias}

1. Del Casale A, Ferracuti S, Rapinesi C, Serata D, Caltagirone SS, Savoja V, et al. Pain perception and hypnosis: Findings from recent functional neuroimaging studies. Int J Clin Exp Hypn. 2015; 63(2): 144-70. https://doi.org/10.1080/00207144.2015.1002371

2. Álvarez E, Uribe K. Hipnosis clínica para mejorar la disposición a la curación de un paciente en la unidad de cuidados intensivos. Enferm. univ. 2016; 13(3): 193-8.

https://doi.org/10.1016/j.reu.2016.04.002

3. Martínez-Montilla JM, Amador-Marín B. La hipnosis terapéutica en la enfermería: Una revisión de la literatura. Hygia de Enfermería. 2017; (95): 27-32.

4. Elsevier BV. NNN Consult. Nanda, Noc, Nic. Elsevier BV; [Actualizado 2019; Consultado mayo 14 2018]. Disponible en: https://bit.ly/2CMzDFE

5. Zahourek RP. Trance and suggestion: timeless interventions and implication for nurses in the new millennium. Holist Nurs Pract. 2001; 15(3): 73-82.

6. Facco E, Pasquali S, Zanette G, Casiglia E. Hypnosis as sole anaesthesia for skin tumour removal in a patient with multiple chemical sensitivity. Anaesthesia. 2013; 68(9): 961-5.

https://doi.org/10.1111/anae.12251

7. Tefikow S, Barth J, Maichrowitz S, Beelmann A, Strauss B, Rosendahl J. Efficacy of hypnosis in adults undergoing surgery or medical procedures: A meta-analysis of randomized controlled trials. Clin Psychol Rev. 2013; 33(5): 623-36. https://doi.org/10.1016/j.cpr.2013.03.005

8. Facco E. Hypnosis and anesthesia: Back to the future. Minerva Anestesiol. 2016; 82(12): 1343-56. [Consultado marzo 14 2018]. Disponible en: https://bit.ly/2HyTsGL

9. Martín-Carbonell MC. Psicología e hipnosis en el tratamiento del dolor. Bogotá: Ediciones Universidad Cooperativa de Colombia; 2017. https://dx.doi.org/10.16925/9789587600780

10. Abdeshahi SK, Hashemipour MA, Mesgarzadeh V, Shahidi-Payam A, Halaj Monfared A. Effect of hypnosis on induction of local anaesthesia, pain perception, control of haemorrhage and anxiety during extraction of third molars: a case-control study. J Craniomaxillofac Surg. 2013; 41 (4): 310-5. https://doi.org/10.1016/j.jcms.2012.10.009

11. Mackey EF. Effects of hypnosis as an adjunct to intravenous sedation for third molar extraction: A randomized, blind, controlled study. Int J Clin Exp Hypn. 2009; 58(1): 21-38.

https://doi.org/10.1080/00207140903310782

12. Antonelli C, Luchetti M, De Trana L. The association of hypno-anesthesia and conventional anesthesia in a patient with multiple allergies at risk of anaphylactic shock. Int J Clin Exp Hypn. 2014; 62(4): 483-91. https://doi.org/10.1080/00207144.2014.931178

13. Wolf TG, Wolf D, Below D, D’Hoedt B, Willershausen B, Daubländer M. Effectiveness of self-hypnosis on the relief of experimental dental pain: A randomized trial. Int J Clin Exp Hypn. 2016; 64(2): 187 99. https://doi.org/10.1080/00207144.2016.1131587

14. Agard E, Pernod C, El Chehab H, Russo A, Haxaire M, Dot C. Apport de l'hypnose dans la chirurgie de cataracte, à propos de 171 chirurgies. J Fr Ophtalmol. 2016; 39(3): 287-91.

https://doi.org/10.1016/j.jfo.2015.04.024

15. Caballero-Muñoz E, Becerra-Salas R, Hullín-Lucay Cosio C. Proceso de enfermería e informática para la gestión del cuidado. Santiago de Chile: Mediterráneo; 2010.

16. Sánchez-Armáss O, Barabasz AF, Barabasz M. Estandarización de la escala Stanford de susceptibilidad hipnótica, forma C, en una muestra mexicana. Enseñanza e investigación en psicología. 2007; 12(1): $131-46$ 